\title{
Bathymetric stripping corrections to gravity gradient components
}

\author{
Robert Tenzer ${ }^{1}$ and Pavel Novák ${ }^{2}$ \\ ${ }^{1}$ School of Surveying, University of Otago, 310 Castle Street, Dunedin, New Zealand \\ ${ }^{2}$ NTIS - New Technologies for Information Society, University of West Bohemia, Univerzitní 22, Plzeř, Czech Republic
}

(Received April 11, 2012; Revised June 15, 2012; Accepted July 2, 2012; Online published August 16, 2012)

\begin{abstract}
To allow for geophysical interpretation of observed gravity gradients, several corrections must be applied. In this article expressions for gravimetric forward modeling of bathymetric (ocean density contrast) stripping corrections to GOCE gravity gradient observables are evaluated numerically. The generic expression for the bathymetric gravitational potential utilizes a depth-dependent seawater density distribution model. The expressions are defined in their spectral representation by means of the bathymetric spherical functions which describe the global geometry of the ocean bottom relief. Numerical examples are given for the bathymetric stripping corrections to selected gravity field parameters computed with a spectral resolution complete to degree 360 of spherical harmonics. All computations are realized globally on the 1 arc-deg geographical grid at the mean satellite elevation of $250 \mathrm{~km}$. The results reveal that the bathymetric stripping corrections to gravity gradients globally vary within $\pm 5 \times 10^{-9} \mathrm{~s}^{-2}$. Extreme values apply mainly along the continental margins where the largest spatial bathymetric gravitation signal variations occur.
\end{abstract}

Key words: Bathymetry, forward modeling, GOCE, gravitational gradiometry, ocean density contrast.

\section{Introduction}

In gravimetric inverse methods for studying the lithosphere structure, the topographic, bathymetric, and additional corrections of all known anomalous mass density structures within the Earth's crust are applied to observed gravity data in order to model the unknown (and sought) density structure or density interface. In geophysics, this step is well known as gravity stripping (e.g., Hammer, 1963). The gravitational field generated by the ocean density contrast (relative to an adopted mean Earth's density) represents a significant amount of the gravitational signal to be modeled and subsequently removed from observed gravity data. Currently available global gravitational models (GGM) as well as global topographic and bathymetric data sets allow for relatively accurate numerical evaluation of topography-corrected and bathymetry-stripped gravity field parameters to a very high spatial resolution.

The GOCE gravity gradiometry satellite mission provides global and homogeneous data sets with well-known stochastic properties. It is thus expected that GOCE gravity field solutions will improve our knowledge about the Earth's inner density structure especially beneath oceanic and continental areas where seismic data are not yet available or their accuracy and spatial coverage is insufficient.

Novák and Grafarend (2006) evaluated numerically external gravitational fields of topographic and atmospheric masses by using spherical harmonic expansions. Tenzer et al. $(2008,2009,2010)$ then computed globally the bathymetry-generated gravity field parameters by using the

Copy right(C) The Society of Geomagnetism and Earth, Planetary and Space Sciences (SGEPSS); The Seismological Society of Japan; The Volcanological Society of Japan; The Geodetic Society of Japan; The Japanese Society for Planetary Sciences; TERRAPUB.

doi:10.5047/eps.2012.07.001 apparatus of the spherical harmonic analysis and synthesis. Novák (2010) computed globally the gravitational potential generated by the global ocean masses with a very high spatial resolution. In these studies a constant value of the ocean density contrast was adopted.

The actual seawater density variations due to salinity, temperature, and pressure typically range within 1020 and $1050 \mathrm{~kg} \mathrm{~m}^{-3}$, with most of this variation being due to pressure (Garrison, 2001). When the actual seawater density is approximated only by its mean value, relative inaccuracies up to about $2 \%$ were estimated in computed values of the bathymetric stripping corrections (Tenzer et al., 2011). Since global bathymetric models are currently available to a very high accuracy and spatial resolution, the errors caused by approximating the actual seawater density by the mean value represent the largest contribution to the total error budget. These errors can reach up to $200 \mathrm{~m}^{2} \mathrm{~s}^{-2}$ and $16 \mathrm{mGal}$ in terms of the gravitational potential and its radial derivative, respectively. The extreme values apply particularly to the computation areas situated over deepest oceans.

Gladkikh and Tenzer (2011) analyzed the oceanographic data of the World Ocean Atlas 2009 (WOA09) and the World Ocean Circulation Experiment 2004 (WOCE04). WOA09 products are made available by the NOAA's National Oceanographic Data Center (Johnson et al., 2009). WOCE04 oceanographic data were provided by the German Federal Maritime and Hydrographic Agency (Gouretski and Koltermann, 2004). They used experimental data of salinity, temperature, and pressure to calculate the seawater density values based on the thermodynamic equation of state TEOS-10 for seawater (Millero et al., 2008). The density values were then used to formulate an empirical model of the global seawater density distribution de- 
fined as a function of the ocean depth to account for density variations due to pressure. The comparison of the experimental and theoretical seawater density values revealed that the new empirical model approximates the above referenced seawater density distributions with the maximum relative error better than $0.6 \%$, while the corresponding average error is about $0.1 \%$. Tenzer et al. (2012a) utilized this empirical seawater density model for computing the bathymetric stripping gravity correction. In this study we adopt this empirical density model to compute accurately the bathymetric stripping corrections to gravity gradient components.

\section{Theory}

Tenzer et al. (2012a) derived the expression for the gravitational potential $V$ generated by the ocean density contrast in the following form

$$
V(r, \Omega)=\frac{\mathrm{GM}}{\mathrm{R}} \sum_{n=0}^{n a x} \sum_{m=-n}^{n}\left(\frac{\mathrm{R}}{r}\right)^{n+1} \mathrm{~V}_{n m} Y_{n m}(\Omega),
$$

where $\mathrm{R}=6371 \times 10^{3} \mathrm{~m}$ is the adopted mean Earth's radius, $\mathrm{GM}=3986005 \times 10^{8} \mathrm{~m}^{3} \mathrm{~s}^{-2}$ is the geocentric gravitational constant, $Y_{n m}$ stands for the spherical harmonic function of degree $n$ and order $m$, and nax is the maximum available degree of spherical harmonics. The 3-D position is defined in the spherical coordinate system; where $r$ is the geocentric radius and $\Omega=(\phi, \lambda)$ denotes the geocentric direction with spherical latitude $\phi$ and longitude $\lambda$. Bathymetric potential coefficients $\mathrm{V}_{n m}$ in Eq. (1) read

$$
\begin{aligned}
\mathrm{V}_{n m} & =\frac{3}{2 n+1} \frac{\Delta \rho_{0}^{w}}{\rho^{\text {earth }}} \\
& \times\left(\mathrm{F}_{n m}-\frac{a_{1} \beta}{\Delta \rho_{0}^{w}} \mathrm{R} \mathrm{F}_{n m}^{1}-\frac{a_{2} \beta}{\Delta \rho_{0}^{w}} \mathrm{R}^{2} \mathrm{~F}_{n m}^{2}\right) .
\end{aligned}
$$

The nominal value of the ocean density contrast $\Delta \rho_{0}^{w}$ is defined as the difference between the reference crustal density $\rho^{\text {crust }}$ and the seawater density $\rho_{0}^{w}$, i.e., $\Delta \rho_{0}^{w}=\rho^{\text {crust }}-\rho_{0}^{w}$. The value of the surface seawater density $\rho_{0}^{w}=1027.91 \mathrm{~kg}$ $\mathrm{m}^{-3}$ (Gladkikh and Tenzer, 2011) was adopted as the nominal seawater density. For the adopted value of the reference crustal density $\rho^{\text {crust }}$ of $2670 \mathrm{~kg} \mathrm{~m}^{-3}$ (Hinze, 2003), the nominal ocean density contrast (at the zero depth) equals $\Delta \rho_{0}^{w}=1642.09 \mathrm{~kg} \mathrm{~m}^{-3}$. The adopted value of the Earth's mean homogeneous density in Eq. (2) is $\rho^{\text {earth }}=5500 \mathrm{~kg}$ $\mathrm{m}^{-3}$ (e.g., Novák, 2010). The parameters of the depthdependent density term in Eq. (2) are: $\beta=0.00637 \mathrm{~kg}$ $\mathrm{m}^{-3}, a_{1}=0.7595 \mathrm{~m}^{-1}$ and $a_{2}=-4.3984 \times 10^{-6} \mathrm{~m}^{-2}$. The numerical coefficients $\mathrm{F}_{n m}, \mathrm{~F}_{n m}^{1}$ and $\mathrm{F}_{n m}^{2}$ in Eq. (2) are defined as (Tenzer et al., 2011)

$$
\begin{aligned}
& \mathrm{F}_{n m}=\sum_{k=0}^{n+2}\left(\begin{array}{c}
n+2 \\
k
\end{array}\right) \frac{(-1)^{k}}{k+1} \frac{\mathrm{D}_{n m}^{(k+1)}}{\mathrm{R}^{k+1}}, \\
& \mathrm{~F}_{n m}^{1}=\sum_{k=0}^{n+2}\left(\begin{array}{c}
n+2 \\
k
\end{array}\right) \frac{(-1)^{k}}{k+2} \frac{\mathrm{D}_{n m}^{(k+2)}}{\mathrm{R}^{k+2}}, \\
& \mathrm{~F}_{n m}^{2}=\sum_{k=0}^{n+2}\left(\begin{array}{c}
n+2 \\
k
\end{array}\right) \frac{(-1)^{k}}{k+3} \frac{\mathrm{D}_{n m}^{(k+3)}}{\mathrm{R}^{k+3}} .
\end{aligned}
$$

The coefficients $\mathrm{D}_{n m}$ of the global bathymetric model (GBM) describe the geometry of the ocean bottom relief. The series $\Sigma \mathrm{D}_{n m} Y_{n m}$ defines the surface spherical bathymetric function $\mathrm{D}_{n}$ of degree $n$. It reads (Tenzer et al., 2010)

$$
\begin{aligned}
\mathrm{D}_{n}(\Omega) & =\frac{2 n+1}{4 \pi} \iint_{\Phi} D\left(\Omega^{\prime}\right) P_{n}(\cos \psi) \mathrm{d} \Omega^{\prime} \\
& =\sum_{m=-n}^{n} \mathrm{D}_{n m} Y_{n m}(\Omega),
\end{aligned}
$$

where $D$ is the ocean depth and $P_{n}$ is the Legendre polynomial. Higher-order terms $\left\{\mathrm{D}_{n}^{(i)}: i=2,3,4 \ldots\right\}$ read

$$
\begin{aligned}
\mathrm{D}_{n}^{(i)} & =\frac{2 n+1}{4 \pi} \iint_{\Phi} D^{(i)}\left(\Omega^{\prime}\right) P_{n}(\cos \psi) \mathrm{d} \Omega^{\prime} \\
& =\sum_{m=-n}^{n} \mathrm{D}_{n m}^{(i)} Y_{n m}(\Omega),
\end{aligned}
$$

where the symbol $\Phi$ denotes the full spatial angle. The components of the gravitational vector and Marussi gravitational gradient tensor, both generated by the ocean density contrast, can be readily derived from the expressions in Eqs. (1)-(7).

\section{Numerical Examples}

The GBM coefficients were used to compute the bathymetric stripping corrections to the Earth's gravitational potential and its first- and second-order derivatives with a spectral resolution complete to degree 360 of spherical harmonics. The computation was done globally on a 1 arc-deg grid at the mean elevation of $250 \mathrm{~km}$. Statistics of all results are summarized in Table 1. The global maps of selected quantities: $V, V_{r}, V_{r r}, V_{\phi \phi}$ and $V_{\lambda \lambda}$ are shown in Figs. 1-3.

The bathymetric gravitational potential $V$, see Fig. 1, is everywhere positive. The maximum signal is over the largest ocean mass concentration (central Pacific Ocean).

The global map of $V_{r}$, see Fig. 2, revealed major structures of the ocean bottom relief. It is everywhere negative, with its absolute maxima distributed over oceanic basins and (partially) subduction zones in Pacific Ocean. The spatial distribution of $V_{r}$ over oceanic areas also clearly revealed the features related to the oceanic divergent zones (i.e., mid-oceanic ridges). It was demonstrated in Tenzer

Table 1. Statistics of gravitational parameters generated by the ocean density contrast computed globally on a 1 arc-deg grid at the elevation of $250 \mathrm{~km}$.

\begin{tabular}{lrrrr} 
Parameter & Min & Max & Mean & RMS \\
\hline$V\left[\mathrm{~m}^{2} \mathrm{~s}^{-2}\right]$ & 16078 & 27173 & 21604 & 2907 \\
$V_{r}[\mathrm{mGal}]$ & -544 & -128 & -309 & 125 \\
$V_{\phi}[\mathrm{mGal}]$ & -287 & 194 & -14 & 77 \\
$V_{\lambda}[\mathrm{mGal}]$ & -221 & 246 & 0.0 & 66 \\
$V_{r r}[\mathrm{E}]$ & -2.0 & 4.7 & 0.9 & 1.4 \\
$V_{\phi \phi}[\mathrm{E}]$ & -2.6 & 2.2 & 0.0 & 0.7 \\
$V_{\lambda \lambda}[\mathrm{E}]$ & -3.5 & 3.1 & 0.0 & 0.7 \\
$V_{r \phi}[\mathrm{E}]$ & -3.5 & 4.1 & -0.1 & 0.8 \\
$V_{r \lambda}[\mathrm{E}]$ & -4.0 & 3.7 & 0.0 & 0.7 \\
$V_{\phi \lambda}[\mathrm{E}]$ & -2.6 & 3.5 & 0.0 & 0.5 \\
\hline
\end{tabular}




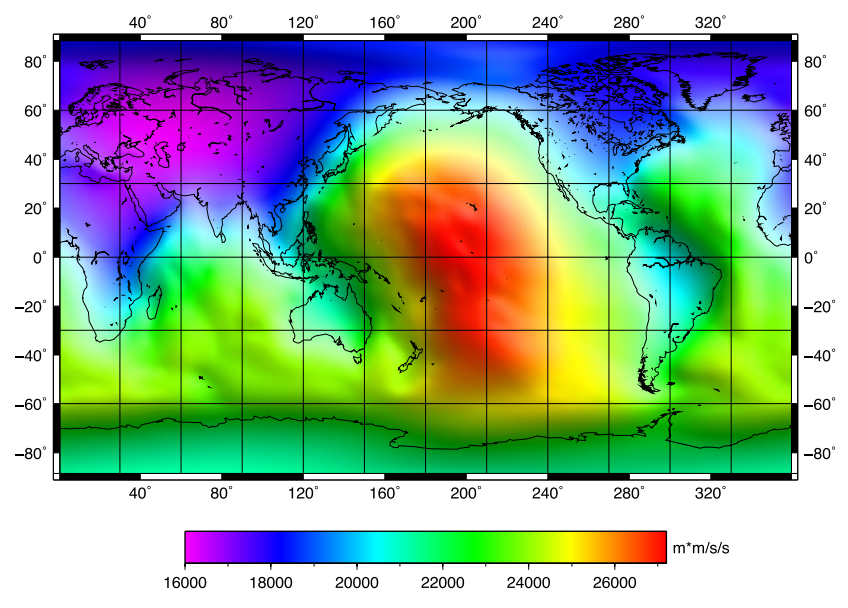

Fig. 1. Gravitational potential $V\left(\mathrm{~m}^{2} \mathrm{~s}^{-2}\right)$.

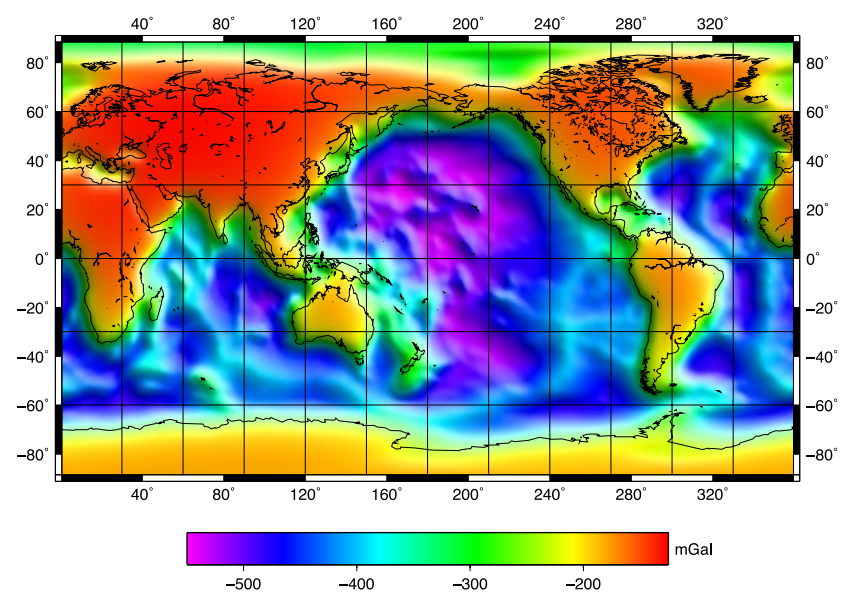

Fig. 2. Gravitational vector component $V_{r}\left(\mathrm{mGal}=10^{-5} \mathrm{~m} \mathrm{~s}^{-2}\right)$.

et al. (2012b), that the application of this correction is essential for the interpretation of the age of oceanic lithosphere (characterized by the formation of oceanic lithosphere along the mid-oceanic ridges, its further speeding and consequent subduction under the oceanic or continental crust along the oceanic subduction zones) which is attributed to mantle convection. The (absolute) values of $V_{r}$ substantially decrease along the continental margins distinctively marking the boundary between the oceanic and continental lithosphere.

Whereas $V_{r}$ is directly correlated with bathymetric depths, the maximum signal of $V_{r r}$ corresponds with the largest spatial variations of the ocean bottom relief. This is seen in Fig. 3(a). The map of $V_{r r}$ revealed major structures of the oceanic lithosphere. The small positive values are found along the oceanic divergent boundaries (mid-oceanic ridges). Over areas of the oceanic abyssal planes, these values further increase. The maxima correspond with locations of the oceanic subduction zones. Boundaries between oceanic and continental tectonic plates are also clearly distinguished (by small negative values) in the gravity map. The bathymetric corrections $V_{\phi \phi}$ and $V_{\lambda \lambda}$, see Figs. 3(b, c), revealed a pattern of the largest horizontal variations of bathymetric gravitational field with respect to the meridional and prime-vertical directions. The maximum positive
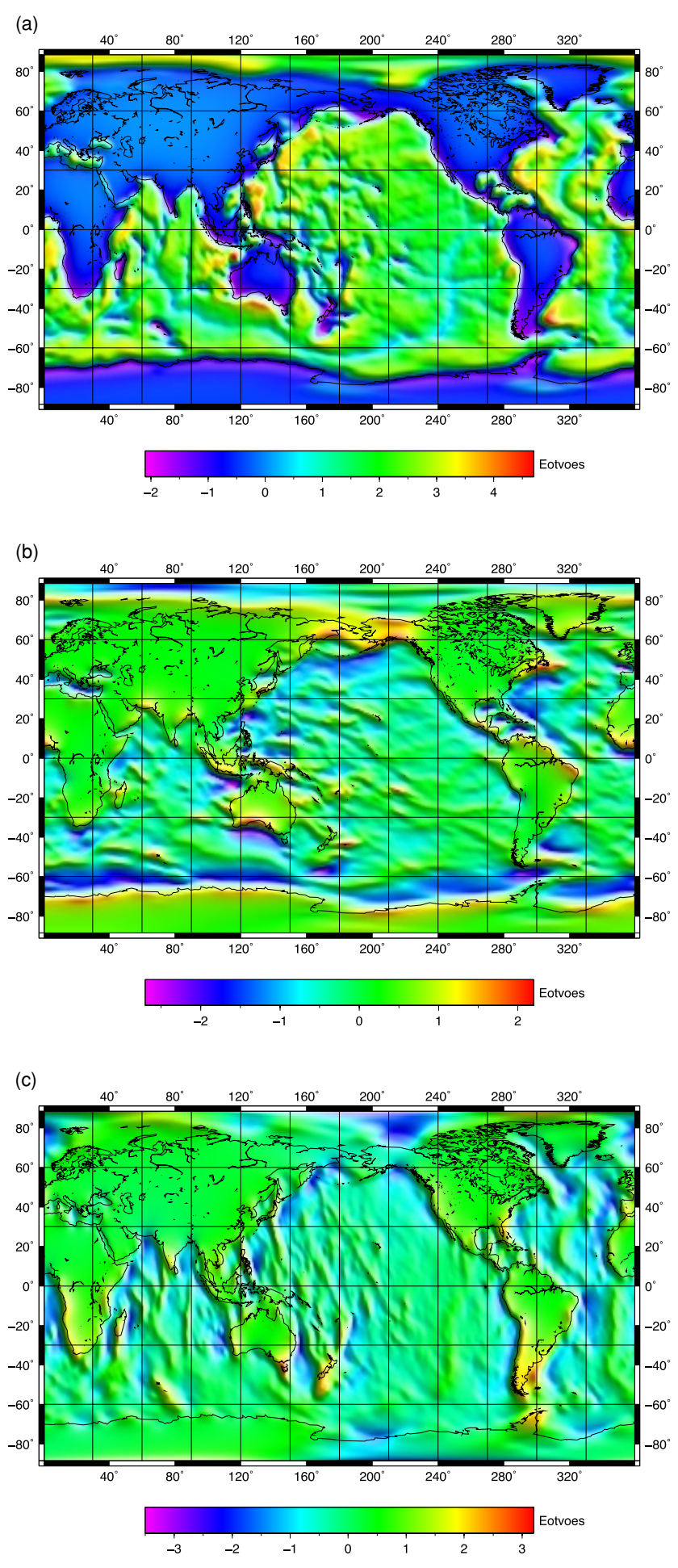

Fig. 3. Gravitational gradient components (a) $V_{r r}$, (b) $V_{\phi \phi}$ and (c) $V_{\lambda \lambda}$ (E $=10^{-9} \mathrm{~s}^{-2}$ ).

and negative values of $V_{\phi \phi}$ are coupled along the continental margins with prevailing latitudinal directions. A similar pattern of the gravity signal is seen in the global map of $V_{\lambda \lambda}$, but in this case with prevailing meridional direction.

\section{Summary and Concluding Remarks}

We have derived and applied spectral expressions for computing the bathymetric stripping corrections to gravity field parameters. The numerical examples were given glob- 
ally on a 1 arc-deg grid computed at the mean satellite elevation of $250 \mathrm{~km}$.

We demonstrated that the maxima of the bathymetric potential $V$ and its radial derivative $V_{r}$ correspond with the largest seawater accumulation in Pacific Ocean. The map of $V_{r r}$ reproduces major structures of the oceanic lithosphere. The spatial distributions of the horizontal components $V_{\phi \phi}$ and $V_{\lambda \lambda}$ are more complex reflecting the largest bathymetric gravitational signal variations with respect to the coordinate directions. Their extreme values are found along the continental margins again with prevailing either south-north or east-west directions. Compared to $V_{\phi}$ and $V_{\lambda}$, the maximum signal of $V_{\phi \phi}$ and $V_{\lambda \lambda}$ along a particular continental margin comprises both, the positive as well as negative values.

Whereas the application of $V_{r}$ is important for modeling and interpretation of the refined gravity field obtained based on analysis of GRACE inter-satellite observables, the bathymetric stripping corrections $V_{r r}, V_{\phi \phi}$ and $V_{\lambda \lambda}$ are applied to GOCE gravity gradient measurements. GOCE gravity gradiometry data provide the accurate information about the Earth's gravity field especially at the medium spherical harmonics (somewhere between degrees 70-200). GRACE data provide the accurate information at the longwavelength part of the gravitational field (up to degree of about 120). The combined inversion of GRACE and GOCE data (including additional geophysical constrains) is thus essential for a more robust and accurate recovery of the Earth's inner density structures.

Acknowledgments. Pavel Novák was supported by the Czech Science Foundation, project 209/12/1929.

\section{References}

Garrison, T., Essentials of Oceanography, Brooks Cole, Pacific Grove, CA, 2001.
Gladkikh, V. and R. Tenzer, A mathematical model of the global ocean saltwater density distribution, Pure Appl. Geophys., 169(1-2), 249-257, 2011.

Gouretski, V. V. and K. P. Koltermann, Berichte des Bundesamtes für Seeschifffahrt und Hydrographie, No. 35, 2004.

Hammer, S., Deep gravity interpretation by stripping, Geophysics, 28, 369-378, 1963.

Hinze, W. J., Bouguer reduction density, why 2.67?, Geophysics, 68(5), 1559-1560, 2003.

Johnson, D. R., H. E. Garcia, and T. P. Boyer, World Ocean Database 2009 Tutorial, in NODC Internal Report 21, edited by S. Levitus, NOAA Printing Office, Silver Spring, MD, 2009.

Millero, F. J., R. Feistel, D. G. Wright, and T. J. McDougall, Deep-sea research, part I: Oceanogr. Res. Pap., 55(1), 50-72, 2008.

Novák, P., High resolution constituents of the Earth gravitational field, Surv. Geophys., 31(1), 1-21, 2010.

Novák, P. and E. W. Grafarend, The effect of topographical and atmospheric masses on spaceborne gravimetric and gradiometric data, Stud. Geophys. Geod., 50, 549-582, 2006.

Tenzer, R., Hamayun, and P. Vajda, Global map of the gravity anomaly corrected for complete effects of the topography, and of density contrasts of global ocean, ice, and sediments, Contrib. Geophys. Geod., 38(4), 357-370, 2008.

Tenzer, R., Hamayun, and P. Vajda, Global maps of the CRUST 2.0 crustal components stripped gravity disturbances, J. Geophys. Res., 114, B05408, 2009.

Tenzer, R., P. Vajda, and Hamayun, A mathematical model of the bathymetry-generated external gravitational field, Contrib. Geophys. Geod., 40(1), 31-44, 2010.

Tenzer, R., P. Novák, and V. Gladkikh, On the accuracy of the bathymetrygenerated gravitational field quantities for a depth-dependent seawater density distribution, Stud. Geophys. Geod., 55(4), 609-626, 2011.

Tenzer, R., P. Novák, and V. Gladkikh, The bathymetric stripping corrections to gravity field quantities for a depth-dependant model of the seawater density, Mar. Geod., 35, 1-23, 2012a.

Tenzer, R., V. Gladkikh, P. Vajda, and P. Novák, Spatial and spectral analysis of refined gravity data for modelling the crust-mantle interface and mantle-lithosphere structure, Surv. Geophys., doi:10.1007/s10712012-9173-3, 2012b.

R. Tenzer (e-mail: robert.tenzer@otago.ac.nz) and P. Novák 